\title{
3 Usage-based Theories of Meaning
}

The central idea underlying this book is that usage-based approaches in the philosophy of language are worthy of being reconsidered for two opposed reasons. On the one hand, recent developments in relevant empirical disciplines clearly support a usage-based approach to semantics-either by being based on usage-based assumptions themselves, or by implicitly assuming such a theory framework. On the other hand, the currently by far most successful theory in that area-Paul Horwich's 'use theory'-is being severely challenged due to its restriction to truth-evaluable content. In simple terms, the most reasonable strategy, therefore, seems to be to take the 'use theory' as a starting point and suggest possible improvements with respect to classes of words (sentences) that hitherto have been hard to handle in that theory (effectively, all classes of words that lack truthevaluable content). ${ }^{1}$

I divided this chapter into two and a half sections. First, I shall introduce the basic idea of accounting for meaning in terms of use (3.1). This also includes a very brief overview of Wittgenstein's private-language argument and Kripke's interpretation thereof, the reason for this being the relationship between acceptance properties and private-language. In 3.2, I briefly explicate Horwich's notion of 'understanding', as that will become highly relevant in the following chapters. The main section, then, is the third (3.3), in which I shall highlight some obstacles to clarifying the claim that 'meaning is use'. As I already mentioned in the Introduction, I reserve the term "use theory" exclusively for Horwich's approach; the umbrella term for his and related theories is "usage-based theories" (and their cognates).

1 The only alternative here is to propose an all-new theory, which for obvious reasons is beyond the scope of this book. 


\subsection{Use}

Famously, the later Wittgenstein is known for having popularised the idea that meaning is use. In the Philosophical Investigations, he says:

One cannot guess how a word functions. One has to look at its application and learn from that. (Wittgenstein 2009, 116e, emphasis original)

All mainstream theories in philosophy that consider themselves 'usagebased' see Wittgenstein as their most relevant predecessor. The common assumption of all these theories is easy to state: meaning is use. The difficulties arise when it comes to specifying what this claim amounts to. I would like to keep this chapter very focused. So there are several things that I will not try to do here. Most importantly, I will not delve into exegetical issues. ${ }^{2}$ Furthermore, I will omit a detailed discussion of usage-based approaches other than Horwich's. For instance, inferentialism (à la Brandom) is a very influential theory of meaning and a prominent exponent of the usage-based camp (Brandom 1994, 2001). However, instead of giving a comprehensive overview of usage-based approaches, I would rather focus on one specific theory in order to discuss some more details. Anyway, Horwich's theory is pretty much in line with my own view that I outline in chapters 6, 7, and 8. Discussing his theory will therefore serve as a backdrop against which I can present my own ideas by explicating important differences. In 3.1.1, I will introduce Horwich's main thesis that the use of a word can be traced back to some underlying acceptance property. Then, in 3.1.2 and 3.1.3, I discuss some critical aspects of this view.

\subsubsection{U Equals A(x)}

The overall use of each word stems from its possession of a basic acceptance property. For each word there is a small set of simple properties which (in conjunction with other factors and with the basic properties of other words) explain total

\footnotetext{
2 I refer the interested reader to the impressive work of Peter M. S. Hacker, which is an invaluable source in this regard (e.g. Hacker 2013).
} 
linguistic behaviour with respect to that word. [...F]or each word, $w$, there is a regularity of the form

\author{
All uses of $w$ stem from its possession of acceptance \\ property $A(x)$,
}

where $A(x)$ gives the circumstances in which certain specified sentences containing $w$ are accepted. (Horwich 1998, 44 and 45 , emphasis omitted)

The basic idea in so-called 'usage-based' accounts of meaning is that the meaning of a given term is-in a sense to be specified-constituted by how the term is used (in a given language community). In slogan form: meaning is use. This, of course, is misleading. Clearly, not all aspects of a term's use can alter its meaning. But the simplified slogan helps to see what is common to all usage-based theories, namely that they all acknowledge that language is first and foremost a means of communication. ${ }^{3}$ And that therefore,

3 This might sound a bit trivial but it has been widely neglected (and denied) in much of the history of philosophy of language. The study of language as a philosophical subdiscipline has always been the study of (context-free) declarative sentences, detached from concrete situations, in which they could possibly be uttered or asserted: 'The mainstream approach to semantics-almost universally accepted amongst linguists and philosophers-is truth-theoretic' (Horwich 2008a, 233, my emphasis). Interestingly, proponents from both camps consider themselves lonesome heroes defending the minority view. For example, Borg $(2004 b, 15)$ begins her discussion of truth-conditional semantics with this:

What kind of thing must an agent know to be a competent language user and what kind of cognitive architecture might lie behind our linguistic abilities? The answer I want to give [...] is not, perhaps, especially fashionable at the moment. For I want to argue for a kind of formal approach to the study of language, which [...] continues through into such approaches as the truthconditional theory of meaning of Davidson et al., and the model-theoretic approach championed by Kaplan and others. (My emphasis)

Philosophically speaking, it is relatively unimportant which side is correct here. Based on the number of publications and prominent current proponents, I have the impression that Horwich is right in this regard. What is more important is that the (real or merely felt) dominance of TCS has left its mark with respect to what people think semantics is all about: context-free specifiable meaning of word/sentence types. This influence is present even in theoretical frameworks that are subsumed under the heading 'usagebased' such as Horwich's use theory or Brandom's conceptual role semantics. In chapter 7, I shall be defending the following two claims: (i) that philosophy of language as an 
roughly speaking, meanings are determined according to how they figure in actual communication situations, i.e. how they are in fact used.

Particular theories differ in the way they cash out the idea that meaning is use. So, in order to get to grips with what is meant by saying this, I shall, by way of example, examine Paul Horwich's view on the matter. This will then serve as a good starting point to develop the basis of a new framework, as Horwich incorporates many correct insights into his theory. Yet, the use theory suffers from some specific difficulties. These difficulties, however, can be handled without omitting the general idea of Horwich's theory. ${ }^{4}$ First of all, Horwich introduces, as a theoretical device, a 'capitalisation convention', according to which the meaning of "dog" is DOG, the meaning of "tea" is TEA, and, generally, the meaning of a term can be referred to by writing the word in capital letters. ${ }^{5}$ It is important to bear in mind that a capitalised word is nothing more than a name. By definition, this name denotes a meaning. If you were asked 'What is the meaning of "dog"?', the answer "DOG" would be perfectly correct. Yet, the capitalisation convention is only a means of metalinguistic description. It goes without saying that one who is able to apply this tool does not, by virtue of this ability, automatically know the meaning of the term to which it is applied. This is the point of introducing the convention in the first place. It enables us theoreticians to refer to specific meanings, irrespective of how they can be individuated.

Writing words in capital letters is a way of referring to meanings, but the interesting question is how to determine them (in principle). Horwich pursues a two-step strategy in this regard. Firstly, he claims that meaning properties are constituted by use properties. Secondly, he then goes

independent discipline is better off if it focuses on actual communication rather than on the corresponding abstractions thereof; and (ii) that language is primarily a means of communication and, hence, its theoretically most interesting aspect is use. For the time being, I take the results of this discussion for granted.

4 The most important resource here, in which the so-called 'use theory' is presented in full detail, is his 1998 book Meaning. Further elaborations of particular objections raised against this theory are collected in Horwich $(2004,2005,2010)$. Horwich's most recent defence of (parts of) the use theory is his 2012 book.

5 In accordance with Horwich's style, I use capital letters to indicate placeholders for meaning-but only in quotes and paraphrases from Horwich. See also the Terminological Preliminaries (1.2). 
on to say that the use properties of a given word are determined by certain acceptance properties associated with sentences in which that word occurs. Actually, Horwich's line of argument is a three-step strategy, for his intermediate thesis is that, ultimately, the meaning of natural-language expressions can be identified with the corresponding concepts $(1998,44)$. Horwich treats the term "means" as approximately synonymous to "indicates". Thus the use of "dog" by a given speaker indicates the presence of (the concept) DOG in the speaker's mind. Mutatis mutandis, for any other word of the speaker's idiolect. The other two steps of this 'three-step account' are exactly the same as in the two-step version. Some avoidable difficulties are incurred by choosing the three-step strategy. This is why I read Horwich as actually defending the simpler picture.

If meanings are concepts, then concepts either play an explanatory role in one's theory of meaning or they do not. If they do, then what one is doing in specifying the underlying non-semantic properties that constitute meanings (read: concepts) is specifying a theory of mental representation (and the origin of these) rather than a theory of meaning proper. Horwich himself suggests this reading by comparing his theory of meaning with, for example, the 'Informational Theory' (see Dretske 1986) or the 'Teleological Theory' (see Millikan 1989) (Horwich 1998, 52). Taken seriously, this amounts to saying that what a term means, in the sense of specifying the underlying properties of meaning, is determined by a theory of mental representation. This, I think, obscures the debate at best. Even if, in the end, utterances of words 'indicate' the presence of corresponding concepts in the speaker's mind, this should be largely independent from one's theory of meaning. Otherwise the question of the underlying (non-semantic) features of meanings is simply moved from the philosophy of language to the philosophy of mind. At worst, this strategy might even be completely misleading, because it is dubious whether there really is such a simple oneto-one connection between concepts and the corresponding words usually used to express them. On the other hand, if concepts play no explanatory role in the theory of meaning, then to identify them with meanings seems superfluous. For even if such an identification may be relevant to make one's theory compatible with certain theories of mental representation, the identification of meanings with concepts, if it is explanatorily insignificant, is irrelevant to a theory of meaning as such. In such a case, it 
seems strange to include the 'identity thesis'-meanings are concepts-into the list of the theory's main principles (as is done in chapter 2 of Horwich 1998).

I shall briefly summarise in somewhat more detail what it means to reduce use properties to acceptance properties. First of all, the notion of 'constitution' applied here means that both properties are co-extensional, but that their corresponding predicates are not synonymous (Horwich 1998, 18-27; in particular p. 25, n. 8). So, to say that meaning properties are constituted by use properties is just a very careful way of expressing the idea that 'meaning is use' or that 'what a word means is determined by how it is used'. The more interesting thing is how use properties can be characterised appropriately. The solution developed by Horwich is unique to his account. It is worth taking a look at how it works in greater detail.

'Use properties' are really only placeholders in the use theory of meaning. The explanatory work is done by those properties with which use properties are identified, i.e. by acceptance properties. Now, what are acceptance properties? The basic idea is this: there is, for each word, a basic acceptance property that can be expressed by a single sentence (maybe a complicated conjunction) which is normally accepted by speakers of a given language and in which the word in question occurs. The acceptance of other sentences in which this very word occurs can be explained on the basis of acceptance of the former sentence. This is why it is called the "basic' use property. Specifying his use of "use", Horwich explains that the use property is

[...] some property of a word type. This property is specified by a generalization about tokens of that type-by the claim that they are all explained in terms of a certain acceptance property, a property specifying the circumstances in which designated sentences containing the word are held true. [...For example,] that we have the disposition to assert "That is red" in the presence of evidently red things [...]. (Horwich 1998, 57-58, emphasis omitted ${ }^{6}$

${ }_{6}$ Please note the apparent similarity between Horwich's way of spelling out acceptance properties and Peacocke's remarks on concept possession: 
Taken together, the main thesis of Horwich's use theory of meaning is that meaning properties (of word types) are constituted by acceptance properties (of the same word types). I will be discussing the postulated close connection between use and acceptance further down. For now, I end the exposition of Horwich's theory and turn to some characteristics of it that might seem problematic. In the process I focus on those features that are of general interest for my own framework. In particular, I shall briefly present Wittgenstein's famous private language argument, as Horwich's explication of 'use' along the lines of 'privately accepted sentences' seems to be at odds with the impossibility of private language.

\subsubsection{The Private Language Argument}

The [use theory of meaning] is focused on the semantic feature of a word. The distinctive form of that feature is that it designates the circumstances in which certain specified sentences containing the word are accepted [...]. For example, it may be that

the acceptance property that governs a speaker's overall use of "and" is (roughly) his tendency to accept " $p$ and $q$ " if and only if he accepts both " $p$ " and " $q$ " [...].

A possession condition for a particular concept specifies a role that individuates that concept. The possession condition will mention the role of the concept in certain transitions that the thinker is willing to make. These will be transitions that involve complete propositional thoughts involving the concept. In some cases they are inferential transitions; in others they are transitions from initial states involving perceptual experience. $(1992,107)$

Which, if applied to an example, gives us that

For a thinker to possess the concept square $(C)[\ldots]$ he must be willing to believe the thought $C m_{1}$ where $m_{1}$ is a perceptual demonstrative, when he is taking his experience at face value, the object of the demonstrative $m_{1}$ is presented in an apparently square region of his environment, and he experiences that region as having equal sides and as symmetrical about the bisectors of its sides [...]. (108) 
[...] Think of all the facts regarding a person's linguistic behaviour-the sum of everything he will say, and in what circumstances. [...] It is not implausible that something like [this regularity is] what explain[s] our overall use of the [word] "and" [...]. (Horwich 1998, 45, emphasis added)

The highlighted passages of this quote indicate that the theoretical scope of the use theory of meaning is to account for the linguistic behaviour of linguistic communities (and thereby to state the meaning of the words deployed by that community) by listing the acceptance properties of the individual speakers. This by itself-the switch from speakers to language communities-may be questioned. It involves some important background assumptions that would need to be justified independently. For instance, one would need to assume that dispositions to accept certain sentences are distributed more or less homogeneously among individuals that belong to one single linguistic community. Also, one would need to allow for differences in dispositions that reflect no corresponding differences in meaning: when, for example, a blind person is not disposed 'to apply "red" to an observed surface when and only when it is clearly red'. 7 All this will be largely ignored in what follows. Instead, I would like to focus on the question: doesn't this fly in the face of Wittgenstein's famous 'private language argument'?

To begin with, the private language argument might be considered problematic for a usage-based account (formulated in terms of acceptance properties) because, as is shown by the above example, acceptance properties are individuated in terms of individual dispositions to accept certain sentences. According to a popular reading of the later Wittgenstein, this can't

7 "Red" is Horwich's favourite example. Note, however, that colour predicates are a particularly difficult class. You might want to argue that the semantics of "red", used by a blind person, differs indeed-even if only slightly-from its ordinary semantics. In this case, replace "red" with a proper name: intuitively, the meaning of a proper name like "Aristotle" can't have a different meaning in the mouth of a seeing person compared with a blind one. Either, then, you still want to maintain the claim that meanings correspond one-to-one to dispositions, which would result in plentiful new meaning postulates (e.g. dispositions of blind people to apply a given term as opposed to allegedly different dispositions of seeing people), or you make sure your dispositional descriptions are as general as to be applicable to seeing and blinded people alike (to name just two groups). Theoretically, disjunctive analysis might be a third, viable option. 
be right. ${ }^{8}$ An individual speaker can't tell a case where he follows a rulesay, to accept " $p$ and $q$ " only when he also accepts " $p$ " and " $q$ "-from the case where it only seems to him as he would follow this rule (although he actually follows a different rule or no rule at all). In Wittgenstein's terms, following a rule and thinking to follow a rule fall into one and the same category in regard to private language. This means that an individual speaker as such would not know whether he follows a 'rule' correctly.

The original argument, in the Philosophical Investigations, is presented in $\S \S 243$ and following. The literature on the topic is fairly independent from the actual source, i.e. the literature on the private language argument is not just exegetical but also, to a large extent, concerned with the content, with the pros and cons of the possibility of private language per se (Stern 2011). This holds in particular for the most famous and most influential interpretation by Saul Kripke in his Wittgenstein on Rules and Private Language (1982). The interpretation of Wittgenstein's work that Kripke offers is sometimes considered to be fairly independent of the original, which is why it usually goes under the name "Kripkenstein". Kripke describes Wittgenstein's worries in terms of dispositions that match past use. The basic idea is that if one takes into account one's whole past use of a given term, then there is no fact of the matter as to whether this term had the meaning that one typically thinks it actually has (now and in the past). This is because there are always many competing rules that accord with past use. Here is Kripke:

Given [...] that everything in my mental history is compatible both with the conclusion that [by "plus"] I meant plus and with the conclusion that I meant quus [i.e. answer " 5 " for all summands greater than 57], it is clear that the sceptical challenge is not really an epistemological one [i.e. rooted in insufficient available data]. It [i.e the problem] purports to show that nothing in my mental history or past behavior - not even what an omniscient God would know - could establish whether I meant plus or quus. But then it appears to follow that there

8 The Wittgenstein works that are most relevant here include his Philosophical Investigations (Wittgenstein 2009) and Remarks on the Foundations of Mathematics (Wittgenstein 1967). 
was no fact about me that constituted my having meant plus rather than quus. [...] There can be no fact as to what I mean by 'plus', or any other word at any time. $(1982,21)$

So, Kripke's worries seem to be even more relevant for Horwich's acceptance-based approach, as there seems to be no way to determine whether any dispositional analysis of the meaning of a given term is ever correct.

\subsubsection{Acceptance in Usage-based Theories}

For our present purposes, I largely ignore exegetical issues. The main aim of this chapter is to give a rough overview of the possibilities and limitations of usage-based theories of meaning. A discussion of Wittgenstein's and Kripke's contribution to the rule-following problem is a good starting point, as there is reason to think that dispositions will figure prominently in any promising philosophical account of usage-based semantics. In other words, there is simply no other reasonable way than to spell out usagebased semantics in terms of the relevant dispositional analyses. Therefore, it will be worth looking more closely at how these two thinkers, Wittgenstein and Kripke, influence contemporary discussions in the field. Specifically, two questions or concerns are relevant in the present context:

(1) Following Kripke, one might want to say that it is impossible to 'read off' particular dispositions that one (supposedly) had in the past from the facts alone, i.e. a given past use of a term is always compatible with a variety of dispositional analyses. In other words, there is more than just one 'correct' interpretation of past use.

(2) Similarly, Wittgenstein emphasises that one can't follow a rule privately, for in that case rule-following and attempting to follow a specific rule would fall into one. This seems to contradict Horwich's approach, for Horwich construes the analysis of core use properties in terms of privately accepted sentences.

Discussing these two 'aspects' of Wittgenstein's criticism will help to illuminate one of the most crucial challenges that today's usage-based theories still face. 
Ad (1): In Horwich's version of the use theory of meaning, use properties are constituted by acceptance properties. In the sense in which water may be identified with $\mathrm{H}_{2} \mathrm{O}$, use properties may be identified with acceptance properties. Acceptance properties, again, are stated in terms of dispositions: they are 'tendencies to accept' specific sentences. Tendencies are dispositions. But then, it seems, Kripke's interpretation of Wittgenstein can be read as a direct criticism of the use theory.

Consider the word "plus". The use theory of meaning tells us that the acceptance properties (disposition) associated with "plus" determine all uses of that word (except occasional mistakes). Suppose, for the sake of argument, that this procedure works in principle. In particular, suppose that if we-linguists, philosophers of language-know the exact acceptance properties of "plus", we would know how the word is used. ${ }^{9}$ The upshot of Kripke's argument, if correct, is that all this is compatible with the claim that "plus" might mean something completely different. Because the adequacy constraint on dispositional analyses is dependent on conformity with the available evidence, which is past applications in this case. The problem here is that, even if the theorems of the use theory are correct in this sense-i.e. if they conform with past verbal behaviour of members of a given language community-a broad variety of competing analyses are also still correct. However the use theorist analyses the alleged acceptance properties that constitute one's overall usage, Kripke could always-given the plausibility of his argument-claim that "plus" might mean 'quus' instead.

To be sure, it may be that "plus" means addition. It is just that we can not know. Even God does not know this, or so Kripke claims. This is my reply: well, maybe we can't tell from our past dispositions that by "plus" we mean addition (now). So Kripke is right in claiming that these past dispositions do not uniquely fix the meaning of the term. But it is an apparent phenomenon that we mean addition by "plus". In order to show this, one may, for example, take a look at how Kripke himself describes the situation:

9 Which is to say that all tokenings of "plus" can be traced back to a person's basic tendency to apply "plus" to such-and-such things in such-and-such circumstances. 
I, like almost all English speakers, use the word 'plus' and the symbol ' +' to denote a well-known mathematical function, addition. $(1982,7)$

This quote is from the opening paragraphs of Kripke's essay. It illustrates quite nicely what Kripke explicitly admits in a later section: that his scepticism in regards to dispositional analyses of usage concerns not our present dispositions but our convictions about the dispositions we had in the past.

What is interesting about this passage is that Kripke is using the only available possibility to formulate one major premise of his argument here: the premise that "plus" in English means 'plus'. The only way to achieve this is by referring to 'a well-known function'. The point I should like to emphasise is that this reveals something important about the status of a 'theory' in philosophy. The 'theory' of meaning, whatever its precise form, is not supposed to show that, say, "plus" means 'plus'. Rather, it is just the other way around. "Plus" de facto means 'plus'-this is the fact the philosophical theory needs to account for. In other words, the question is not, which dispositional analysis is correct (irrespective of whether it concerns past or present use) but why a particular one is correct. To be sure, this view per se is not sufficient to argue against Kripke's Wittgenstein or Quine's (1960) inscrutability of reference. My point here, rather, is that, given one's specific intellectual interests-e.g. designing an empirically validated, philosophically informed theory of meaning-certain problems can simply be bracketed out. Given that we want a theory that fits with our pre-theoretic assumption that "plus" means 'plus', we do not need to consider scenarios in which it meant 'quus'. Given that we want a theory that fits with out pre-theoretic assumption that "rabbit" refers to rabbits, we do not need to consider scenarios in which it referred to undetached rabbit parts.

Ad (2): A related obstacle, which, in particular, applies to the specific variant of usage-based semantics defended by Horwich, is Wittgenstein's observation that one can't follow a rule privately. The way Horwich formulates his idea of 'core use properties' sounds as if his account could fall prey to the Wittgensteinian objection:

The regularities of use that (I am suggesting) constitute the meanings of words concern the circumstances in which spe- 
cified sentences are privately accepted (i.e. uttered assertively to oneself). (Horwich 1998, 94, emphasis omitted)

The gist of Wittgenstein's private language argument is that 'private' rulefollowing is not rule-following after all. Proper rule-following requires a corrective; e.g. a language community that 'sanctions' obvious deviant behaviour. According to the Wittgensteinian argument, one can't privately accept sentences and thereby conform to a particular regularity. Applied to the use theory, the argument amounts to saying that there can't be regularities like Horwich's 'tendencies' that explain the overall use of a given word by a given speaker. This is because all competing dispositional analyses could be just as correct.

It is not exactly clear what the private language argument is an argument against. In a natural reading, it is an argument against the possibility of a 'private language', where this is a language of a single individual. This affects the quoted aspect of Horwich's theory only insofar as we conceive of it (i) as being concerned with the meanings of words as they are used by individual language users and (ii) as referring only to individuals when it comes to meaning constitution. Neither condition is met in the present case. Firstly, although the quoted passage suggests an individualistic reading, it is clear from the outset that the use theory of meaning is a general theory about natural language. It is not directly concerned with idiolects (only to the extent that they affect semantics within language communities). A fortiori, it is not concerned with languages independently from the communities in which they are embedded. It is, if you like, merely a stylistic choice to state the properties that constitute the use of a given word in terms of individual dispositions. This does not affect the fact that the theory applies only to complete natural languages, and, hence, its adequacy is measured relative to them. One idea behind the 'individualistic' formulation of Horwich's dispositional analysis is surely the following. There is a very close link between individual tendencies to accept certain sentences and meaning in language communities, because the relevant dispositions are clearly shared by a significant proportion of people. In this respect, it makes sense to say that meaning is constituted by individual tendencies (had they been different, the relevant meaning would have been different as well); but the meaning to which this analysis applies is still meaning 
in communities. Within a community, people share particular tendencies such as the tendency to apply "red" to red objects.

A second relevant clarification in this context is the following. Although the sentences Horwich talks about are privately accepted sentences, the relevant tendency is determined and corrected by the language community that the person who accepts sentences participates in. So, although it is surely correct, and in fact quite accurate, to say that the basic disposition that constitutes my overall use of the term "red" is my tendency to apply it to red objects, my motivation behind this use-i.e. the corrective at play here-is that other members of my language community have a similar tendency. From this point of view, Horwich's dispositional analyses in terms of privately accepted sentences concern language use in communities. For this very reason, Horwich's appeal to privately accepted sentences is immune to Wittgensteinian private language objections. In a similar fashion, Kripke himself notices that

What is really denied [with the private-language argument] is what might be called the 'private model' of rule following, that the notion of a person following a given rule is to be analyzed simply in terms of facts about the rule follower and the rule follower alone, without reference to his membership in a wider community. (Kripke 1982, 109)

Applied to the use theory of meaning, this amounts to the following: meanings are constituted by individual dispositions to accept certain sentences. These dispositions, in turn, are influenced by the language community in which the individual speaker participates. ${ }^{10}$ In this sense, the private language argument is no threat to the use theory of meaning, since it is a the-

${ }^{10}$ In the passage quoted above, Kripke is dealing with meaning ascriptions, rather than meaning itself. But there is an immediate link between our actual ascription practice and meaning constitution. Due to this link, Kripke's remarks are immediately relevant also for meaning constitution. If a given community ascribes certain meanings to the words used by a given speaker whenever he is following a particular rule (and refrains from doing so otherwise), then the person in question, if he wants to be understood, will try to conform to the behavioural expectations of others. For example, when the language community expects me to apply "red" only to evidently red things (under appropriate conditions), and I recognise this expectation, then I will-on the whole-make sure that I apply "red" accordingly, i.e. I will, normally, apply it only to red things. The mean- 
ory about language communities. This ensures that individual speakers $d o$ not privately follow rules (i.e. conform to specific dispositions), although they privately accept certain sentences.

In light of this specification, the problem with Horwich's core use properties-as far as the private language argument is concerned-seems to be that it invokes an unjustified individualistic reading of his examples. However, this weakness might be repaired quite easily by emphasising the two issues that I just mentioned. Given these, one could say that the meaning of a word is determined by the set of acceptance dispositions that an individual speaker has with regard to certain sentences in which that word occurs. Words have meaning only relative to language communities: in particular, the respective sets of dispositions are, on the whole, distributed homogeneously among a community. They are accommodated by speakers according to the behavioural expectations of others. Of all possible word meanings that are in accordance with the set of 'compatible' dispositions (e.g. 'plus' vs 'quus'), a word de facto has just that meaning that is also in accordance with the ascription practice within the relevant language community. This last proviso answers Kripke's worry that there might be competing dispositional analyses that accord with past use. However, Kripke himself acknowledges that in regard to present usage we all seem to agree that by "plus" we mean 'plus' (as opposed to 'quus'). If that is correct, then, by the same token, the same is true of past use-since we all agree that we meant 'plus' in the past. The sceptical philosophical reply-'You can't know for certain that this is the correct interpretation of your past dispositions!'-is inadequate here, for the alleged 'epistemological' problem is a rhetoric trick. We know which interpretation of past applications of "plus" is correct (just as we know which interpretation is correct concerning present use); so, if Kripke's argument indeed shows that this fact can't be captured by certain dispositional analyses, then there is indeed a need to improve the relevant analysis. But there is no need to worry whether our interpretation, on which we agree anyway, was correct in the first place.

ing ascriptions of other participants of a language community thus constantly influence one's dispositions to apply certain words. 
Independently from the plausibility of construing a usage-based theory of meaning in terms of acceptance properties, in this section it has been shown that such an account can be defended against objections that focus on the private language argument. Basically, it is just a matter of precise formulation. A clear answer to the question whether private-language arguments are capable of undermining the plausibility of Horwich's theory depends on the exact formulation of acceptance conditions. If it is appropriately relativised to linguistic communities, roughly like in the above formulation, then the argument does no harm to the theory. Nevertheless, this intermediate result leaves it entirely open at this point whether there could be any further problems associated with acceptance-based approaches in general. I return to this question in section 6.2.2.

\subsection{Understanding}

"Understanding" is a notoriously vague term. In Horwich's theory, it means to have implicit knowledge of the use associated with a given term. I adopt this view in what follows. Therefore, I shall discuss this notion at some more detail in this section.

\subsubsection{Understanding and Knowing the Meaning}

Now I should like to look in detail at how a use theory in the spirit of Horwich's can be combined with the apparent conceptual equivalence between 'knowing the meaning of $x$ ' and 'understanding $x$ '. Both concepts are closely linked, and examining their exact relationship will reveal important aspects of the notion of 'understanding', which figures prominently in usage-based semantics in general, and in the 'use theory' in particular.

'Knowing the meaning of a term' implies understanding it. To see this, it is crucial to first look more closely at the two involved notions, "knowing (a meaning)" and "understanding", respectively. Horwich's suggestion, which I shall adopt in the following, is that to 'understand a term' is to know how it is used. For instance, if the relevant term is in my active vocabulary, I know how to use it, or else, I would know how other speakers use the term. 'Knowing how to use a term' implies, in turn, that one knows the term's inferential role; that one's use is roughly in accordance 
with the established use within the corresponding community (i.e. that it is correct) ${ }^{11,12}$ that one is able to answer appropriately if asked for the meaning of the term; and maybe a bit more, depending on the theory-specific notion of 'use' involved.

To state when exactly somebody can be said to "know the meaning of " $x$ "' is slightly more complicated. On a very natural, albeit somewhat naïve, reading, it might mean that one is simply able to state the meaning of " $x$ ". This is surely one important aspect. True as it is, without further restrictions this is essentially valueless. To name but one problem here, whether statements are appropriate meaning statements depends crucially on the semantic theories considered. In general, there at least as many permissible variants to state the meaning of a given terms as there are semantic theories. What is required here is a theory-independent notion of semantic knowledge. Such a theory-independent conception is likely to be pretty similar to the description of 'understanding' explicated above. Because knowledge of meaning involves essentially knowledge that manifests itself in observable verbal behaviour: which inferences one draws from which sets of premises; which answers one gives to certain questions; how one behaves non-verbally in response to others' behaviour; and so forth. Pressed in this direction, one easily gets the impression that understanding is indeed very similar to semantic knowledge, and that the only thing that is undoubtedly very dissimilar is explicit knowledge of the underlying prop-

${ }^{11}$ In this sense, I agree with Horwich that understanding comes in degrees (see Horwich 1998, 17-18).

12 The idea that understanding a term implies that a speaker's use of that term is roughly in conformity with how the word is used within her language community (or by 'the experts') goes back to Putnam (1975). He discusses this restriction in the context of reference fixing. Horwich expands this restriction and relates it to meaning (as opposed to mere reference); he sees agreement (of use) with the group of 'experts' as a measurement of understanding. In general, a person fully understands a given term when his use is 'correct' in the sense that it is in accordance with the experts' use. The other end is marked by the threshold at which a person can be said to use the same word type as his language community. It is a difficult matter to determine where exactly to drawn the line here. If someone uses "tiger" and does not know that a tiger is an animal, this would surely be below the threshold (Putnam 1975). Still, the threshold for what is appropriate might in fact be substantially low, so that, if one is asked what the meaning of "democracy" is, "a kind of regime" might already count a sufficiently informed answer (in the sense that one 'talks about the same'). 
erties of meaning. In other words, the expression "knowledge of meaning" in the former sense, in which every competent language user knows the meaning of the terms that he masters, is by its very nature tightly linked to the use associated with the term, i.e. tightly linked to understanding.

\subsubsection{Horwich on Understanding}

The niceties that are relevant in the area of understanding are worth discussing, since they reveal important aspects of Horwich's use theory. So just like before, when Wittgenstein's rule-following argument served as the starting point for the discussion, understanding and knowledge of meaning will now be vehicles by which I would like to present some details of the theory at stake. The most problematic aspect of Horwich's theory in the context of 'understanding' surely is the so-called capitalisation convention. Because as has already become clear above, it is quite 'easy' to tell the meaning of a word (in a very weak sense of "telling"). If really only naming is concerned, it is sufficient to master the convention. The tension between his account and the closely connected concepts of knowing the meaning of a term and understanding it forces Horwich to admit some ambiguity when it comes to what it is to 'know the meaning of a term'. Officially, the meaning of "dog" is just DOG, which in turn is specified in terms of a use property $u$. However, since no understanding of "dog" is required in getting there, this can't be the whole story, of course. Accordingly, in the passages dealing with understanding, Horwich specifies his notion of 'knowing the meaning of a term' in such a way as to directly referring to use properties, instead of taking the route via meaning properties:

The degree to which an individual understands a word is constituted by the degree of similarity between what it means in his idiolect and what it means in the communal language. And this degree of similarity in meaning is in turn constituted by the degree of similarity between the explanatorily basic use property, $u(x)$, that determines the word's overall deployment in the community and the use property that determines its deployment by the individual. (Horwich 1998, 17-18) 
These facts concerning use properties are known only implicitly, Horwich claims. This allows him to say that somebody can understand a given term, without being able to explicitly state more than the obvious fact that "dog" means DOG, which, in principle, is knowable independently from understanding.

But there is a problem lurking here, because in Horwich's account, evidence to the contrary notwithstanding, 'understanding' and 'knowing the meaning' must fall apart. Horwich seems to admit that there is a very close connection between the two, in particular that, as we assume, knowledge of meaning implies understanding (no matter whether the reverse holds). He claims that 'those who do not understand the word (i.e. do not know what "dog" means) ... [do such and such]', and that there is a 'conceptual equivalence of "understanding a word" with "knowing what it means"' (1998, 16 and 18, respectively). So, in a way he treats both notions as synonymous or approximately synonymous. It makes sense to take Horwich as having the everyday notion of semantic knowledge in mind here. This is, as I said above, distinct from the knowledge associated with the constitutive properties of meaning.

In Horwich's account, almost all knowledge concerning meaning that he discusses in his work is implicit knowledge. The only meaning facts explicitly known (if any) are those about capitalised expressions, i.e. what a speaker might know explicitly is the name of the meaning of a given term, but not the meaning itself. This allows him to equate understanding with knowledge of meanings: investigation into meanings (for example, by analysing uses in order to list relevant acceptance properties) does not affect one's understanding. Understanding varies with the degree of similarity between one's own use of a given term and how it is used within the corresponding community-and so does knowledge.

So there are at least two senses in which one can be said to know the meaning of a given term. One is the usual, implicit understanding of the notion. On the other reading, 'knowledge of meaning' concerns the metalinguistic knowledge philosophers and linguists try to gather when they examine the constitutive basis of semantics. It seems plausible that the former notion can't be dissolved from the notion of "understanding", for, as already indicated above, they share a common evidence basis: use. In a usage-based understanding of meaning, then, use is the commonality 
between the two notions. If I investigate the use that constitutes the semantics of my own language, I explicate-provided I work accurately-a body of information that I already knew implicitly before.

There is just one problematic aspect of Horwich's theory that I would like to mention in the present context. His favourite example of a meaning statement that is based on acceptance properties is this: 'that our use of "red" can be explained in terms of "the disposition to apply "red" to an observed surface when and only when it is clearly red' (Horwich 1998, 45). This itself is surely correct, or at least very close to the truth. But the example term "red" oversimplifies things here, since its meaning can be analysed-as the example illustrates-largely independently from the meaning of related terms. Semantic holism in its simplest form is the claim that the meaning of a given term is dependent on (or can't be stated independently from) the meanings of other terms that are 'linked' to it (cf. Devitt 1993). The idea here is that language is a 'semantic net' into which all meaningful expressions are embedded. In this view, the meaning of a term changes as soon as any other term in the semantic net is altered (provided they are linked appropriately). Holism is a relatively old idea; its most influential proponent in 20th century philosophy was probably Quine, who famously claimed 'that our statements about the external world face the tribunal of sense experience not individually but only as a corporate body' $(1951,38)$. Today, there is a variety of different distinctions in the area of holism, none of which, however, is particularly relevant to my point here. "Red", being a colour term, is a word whose meaning is-even if semantics is a holistic enterprise-explicable more or less independently from what related terms mean. This, in turn, means that the 'knowledge of meaning' involved here is in fact quite self-contained. Other examples will probably turn out to be far more complicated than this one.

By way of summary, it seems-in light of the previous discussion-reasonable to define "knowledge of meaning" and "understanding" as follows. A person knows (explicitly) the meaning of a given word iff that person is able to state the circumstances in which that word may be applied in conformity with the standards of the respective language community. A person understands a given word iff that person knows its use, i.e. she knows how to use it 'correctly' herself (ability of correct application) or how it is used by others (ability of correct interpretation). This knowledge includes: 
(i) knowledge of the inferential role of the word; (ii) the ability to use the word roughly in accordance with how the word is used within one's language community; and (iii) the ability to answer (more or less appropriately) questions regarding the meaning of the word.

\subsubsection{Understanding and Knowledge Covary}

The above discussion, hopefully, shows two things. Firstly, that understanding and semantic knowledge are indeed tightly linked notions. Secondly, that there are several readings of 'knowledge of meaning' at work. Let me summarise the argument by recapitulating these two issues.

The phrase "knowledge of meaning" is ambiguous, it seems, in several respects. One important differentiation proposed by Horwich is between implicit and explicit knowledge. This, however, is problematic insofar as it presupposes a theory-bound understanding of the body of information that is implicit (e.g. knowledge of conceptual role). I think the direction of Horwich's line of argument is basically right, but it should better be formulated in epistemological terms. The implicit knowledge concerning meaning simply corresponds to 'understanding'. Understanding is the ability to employ one's implicit knowledge of applying or interpreting particular words, sentences, theories, and so on. This connection seems independent from the semantic knowledge itself. For instance, it could consist of (knowledge of) Tarskian truth conditions. Explicit semantic knowledge is knowledge about the constitutive basis of meaning: e.g. knowledge that the meaning of "red" is essentially determined by a tendency to accept the sentence "That's red" in the presence of red objects, or knowledge that "red" refers to a specific colour. Irrespective of one's favourite theory of meaning (i.e. irrespective of whether the postulated explicit knowledge gets formulated in usage-based terms), the touchstone of both implicit and explicit semantic knowledge is observable behaviour. Understanding is an ability, as I just said. Abilities are ascribed on the basis of what people who seem to have this ability in fact $d o$.

So, what Horwich does when he claims that he only makes explicit the implicit knowledge that every competent language user has anyway is skipping one step in the above argument. He would need to claim that the explicit knowledge that he postulates (e.g. sets of acceptance proper- 
ties) best explains the behavioural patterns that competent language users show and which we take as evidence for their ability to understand. Summing this up in simple terms, use is not the common factor of implicit and explicit semantic knowledge but it is the common factor in an epistemological sense, namely in the sense that the very same behavioural patterns that justify ascriptions of understanding also justify assumptions concerning a specific constitutive basis of meaning.

In light of these considerations, the link between understanding and knowledge of meaning seems obvious. Understanding is just implicit knowledge of usage-based facts, i.e. knowledge of how words (and the like) are applied and interpreted. One's ability to understand a term is reflected in one's overall linguistic (and non-linguistic) behaviour, but it is constituted by one's implicit knowledge of the use properties of that word. Given this, Horwich's proposal for 'measuring' understanding in terms of the similarity of one's use of a term with the overall employment of that term in the corresponding linguistic society seems quite reasonable. ${ }^{13}$ For this allows us to say, independently of any theory, some people understand certain words better than others.

\subsection{Explicating the Claim}

The basic idea behind usage-based semantic theories is that 'meaning is use'. This, to be sure, is a motto or slogan at best. So, one step toward a semantic framework whose value might be assessed appropriately is to make clear what this claim amounts to, once it is spelled out. This is what I would like to do in this section.

\subsubsection{Core Use Properties}

One argument against usage-based accounts of meaning focuses on the apparent fact that if meaning were use (as such), then with every change in

\footnotetext{
${ }^{13}$ Provided the involved background assumptions are plausible. In particular, one would need to justify the assumption that complete understanding consists in perfect compliance with the overall use in a given society.
} 
use, a word would alter its meaning. ${ }^{14}$ This is an undesired consequence, for it is contrary to our intuitions. Intuitively speaking, not every new instance affects the meaning of a term. Suppose, just for the sake of argument, that we spell out use properties in terms of acceptance properties. In 2012, one would accept the sentence "Dr Angela Merkel is Germany's current chancellor". A few years later, this sentence wouldn't be accepted any more. Now, if the meaning of "Dr Angela Merkel" depends on all its uses (instantiations of the relevant type) and, hence, can be explained by listing all accepted sentences in which that term occurs, then its meaning has changed somewhere around the time of Merkel's de-selection as chancellor. Rather than accepting this radical result, we would want to explain the difference in use in terms of different facts in 2012 and, say, 2017.

Irrespective of whether one wants to define use in terms of acceptance properties, one needs an answer to this problem. Somehow, therefore, I need to restrict the scope of "use" in my definition of "meaning". On the other hand, I certainly should allow that even small changes in use 'result' in altered meanings. The most obvious case is in which the change is based on a new definition of the term. I shall illustrate this by the example of "planet". On planets, Horwich writes that

[...] if a planet beyond Pluto were discovered, and we started to say "There are ten planets", we would not thereby have given the word "planet" a new use. [...] [The opponent will then ask in reply], what is the basis of the distinction that is being assumed here between the use facts (like, perhaps, our disposition to accept "Planets orbit stars") which could plausibly be held to constitute the use of "planet", and other use facts (like our disposition to accept "There are nine planets") which surely could not? $(1998,59-60)$

\footnotetext{
${ }_{14}$ The details depend on the particular theory. For example, according to Horwich's account, a word would-if the objection were correct-change its meaning if the change in use resulted in a corresponding change of the list of accepted sentences (in which that word occurs). See Horwich (1998, 59-60) for a-very short-reply to this objection. The basic idea is to restrict the list of accepted sentences to those necessary to account for all sentences in which the particular word occurs.
} 
There may be other reasons to decline acceptance of "There are nine planets" than the discovery of a tenth planet. In 2006, the definition of "planet" was modified, so that according to the new definition, Pluto is not a planet any more. ${ }^{15}$ This, of course, altered 'our disposition to accept "There are nine planets"' (now there are eight planets in the solar system). Horwich is surely right in noting that our disposition to accept "There are nine planets" is, if you like, an inessential aspect of our use of "planet". However, we are certainly inclined to say that our altered dispositions in the case of redefinition is due to a change in meaning. Redefinitions are paradigm examples of altered meanings.

While Horwich's example shows that acceptance properties may differ with constant meaning, the redefinition of "planet" illustrates that, in regard to the very same acceptance properties, altered dispositions may also be the result of corresponding changes in meaning. This in itself does not undermine the claim that this disposition does not constitute the use of "planet". But it shows that what makes this a case of irrelevant dispositions is not-as is suggested by the example-that an empirical observation was involved. It is true that such observations do not directly affect meaning. ${ }^{16}$ This, however, is not what sets these dispositions apart from others like the disposition to accept "Planets orbit stars". Horwich's point is that he wants to reanimate the analytic/synthetic distinction: our disposition to accept "Planets orbit stars" is left unaffected by any minor redefinitions (e.g. the redefinition that excludes Pluto) or empirical observations. In other words, "Planets orbit stars" is an analytic truth about planets and therefore our disposition to accept this sentence is among the essential dispositions that constitute our use of "planet". The basic idea here is that as long as the analytic truths associated with a given word remain, its core use properties remain the same as well.

It goes without saying that there are alternatives to the Horwichian approach. For example, instead of positing one word type "planet", one could acknowledge that there were two different words, before and after 2006.

\footnotetext{
${ }^{15}$ See 'IAU 2006 General Assembly: Result of the IAU Resolution votes', http: // www. iau.org/public \_press/news/detail/iau0603/, accessed on: 18/06/2012.

${ }^{16}$ Yet they might do so indirectly, as new observations might make a redefinition of terms necessary.
} 
This, however, would lead to further consequences beyond the scope of this chapter. Many words alter their meaning more or less continuously; compare, for instance, the semantic 'stability' of words like "because/'cuz" or "war" on the one hand and "Aristotle" or "twenty-three" on the other hand. It certainly seems implausible to posit arbitrarily new types here. Accordingly, I shall leave all this aside and focus on the theoretical motivation for core use properties, rather than on their exact design.

Core use properties are, in fact, essential to any usage-based theory of meaning. The mere claim that 'meaning is use' is so abstract and general that, if it were true, it were vacuously true. ${ }^{17}$ One respect in which the claim that 'meaning is use' must be specified concerns the apparent fact, discussed above, that primary meaning-or its theory-specific equivalentplays an important role in a variety of linguistic effects such as irony, metaphor, implicature, etc. For example, the potential of "It's nice weather today" to mean 'The weather is quite bad' in certain circumstances seems to be parasitic on the quite different potential of this particular phrase to mean 'It's nice weather today' in most other circumstances (what is commonly called 'under standard conditions'). Now, if one takes the claim that 'meaning is use' all too seriously, then the problem is that the use of a term simply seems to be the totality of all word type instantiations, among them instances of, say, "nice" that mean 'bad', or 'interesting, or 'misplaced', or what have you. Yet, the potential of "nice" to mean 'bad' seems to be rooted solely in the fact that "nice" primarily means 'nice', and nothing more. In other words, usage-based theories of meaning are required to systematise use in the sense that they need to distinguish a core of use properties that accounts for use tout court (e.g. the core use property that constitutes the potential of "nice" to mean 'nice').

More generally, semantics is not just the study of meaning of naturallanguage expressions but also of abstractions thereof. Accordingly, semantics not only explains that and why, for example, "nice" might mean 'nice' in a particular situation, and 'bad', or 'interesting', or 'misplaced' in others. It also explains (or should at least be compatible with accompany-

\footnotetext{
${ }^{17}$ Even proponents of truth-conditional semantics or formal semantics potentially agree that use plays some role in determining meaning-e.g. they might allow that the typedifference between "bank", referring to an institution, and "bank", referring to a special parts of rivers, is rooted in differences in use.
} 


\section{Usage-based Theories of Meaning}

ing frameworks that explain) why the interpretation 'nice' is a particularly relevant one and why other interpretations are derivative or peripheral. The idea here is that core use properties can serve as a basis for explanations that generalise over the totality of use. In Horwich's theory, core use properties are identified with distinct acceptance properties, where the latter are stated in terms of dispositional analyses: the core use properties of a given term can, so argues Horwich, be stated by specifying a (singleton) set of sentences which a competent language user would accept under appropriate conditions. For instance, a competent user tends to accept "That's red" in the presence of red objects. This explains his overall employment of the term "red". In this sense, the 'theory' is able to account both for literal as well as peripheral, non-literal word-type instantiations.

The list of sentences the acceptance of which constitute one's (core) use of a particular word needs to be restricted somehow. As should be clear at this point, sentences involving empirical claims certainly need to be excluded; that is, those sentences the acceptance or rejection of which can be explained away by referring to empirical facts are inessential to meaning constitution. In the first example, the acceptance of "Dr Angela Merkel is Germany's current chancellor" depends on whether Merkel is taken to be the current German chancellor. Similarly, for the first "planet" example. In the case of a redefinition, there is no empirical fact (in the relevant sense) that decides the matter. In other words, there must be some non-empirical facts that distinguish essential dispositions from inessential ones. Or, in Horwich's own words: '[...] the way to pick out the particular use property of a word that comprises what we call "the use" is to find the use property that provides the best explanation of all the others' (Horwich 1998, 60). Far from being an answer, this is more like a re-description of the initial problem.

An obvious alternative would be to bite the bullet and accept that the use of a word really is the totality of its use. This option, however, runs counter to our intuitions. Consider the redefinition case again: "planet" changed its meaning and we modified our use of it ('How many planets are there?' 'Eight!'). But this is not the whole story. We, language users of English, have accommodated our use just because the meaning of "planet" has changed. And this is incompatible with the claim that the total use of "planets"-including our disposition to accept "There are eight planets"- 
constitutes its meaning. The interim conclusion, then, is something like this: on the one hand, I can't consistently maintain that the total use of a term constitutes its meaning; on the other hand, there is no conclusive solution yet as to where to draw the line between the dispositions considered essential and the rest. ${ }^{18}$

Another, admittedly less obvious alternative is to give up the idea that (explanatory basic) use properties of a word can be individuated on the basis of acceptance properties. This is because the whole problem concerns only the quite specific issue of how to separate the use-constituting sentences from the class of all accepted sentences. If I would omit acceptance properties, I could also simply ignore the corresponding dispositions. This solution has one further, important advantage. I have already mentioned the problem that a usage-based account that focuses merely on acceptance properties leaves out the huge class of truth-valueless sentences (or words that do not contribute truth-evaluable content). Can we reasonably say that in such-and-such circumstances sentences like "Hello!" or "What the $\mathrm{f}^{* *} \mathrm{k}$ ?!” are accepted? The words occurring in these sentences surely have their respective meanings, and it is likely that these are determined by how the words are used as constituents in, inter alia, the cited sentences. But you can't accept "Hello!" in appropriate situations in a similar way that you accept the application of "red" in the presence of an object that is clearly red (if you do). Accordingly, in light of the problems facing 'acceptance-based' accounts, I suggest spelling out use properties not in terms of acceptance properties but somehow more broadly (see chapter 6).

This strategy serves two distinct purposes. Firstly, it leaves open the possibility to generalise one's theory of meaning to truth-valueless sentences and words occurring in them. Secondly, it sets issues of meaning apart from the discussion of the analytic/synthetic distinction. The former is a welcome result because it frees me from the need for yet another theory that would then account for truth-valueless sentences. The latter is a

${ }^{18}$ The solution that I suggest in part three of this book is to distinguish between semantic descriptions and constitutive bases of meaning. The latter (total use) leaves the relative 'stability' of the former (dispositional analysis) intact, as it were. Typically, when we are talking about 'meaning', what we mean is semantic descriptions, which is why the intuition that total use does not affect meaning directly is indeed correct. 
virtue because this is an unsettled debate, and you better play safe and not base your theory of meaning on such a controversial distinction.

\subsubsection{Linguistic-Philosophical Terminology}

Several disciplines contribute to the project of a 'theory of meaning', among them philosophy of language, philosophy of mind, some branches of linguistics, anthropology, cognitive psychology, etc. They all differ in their theoretical aims and with respect to the 'methods' they employ. When it comes to broad classifications of semantic theories, several distinctions are drawn, of which the distinction 'truth-conditional approaches vs usagebased approaches' is just one. ${ }^{19}$

The term "usage-based" figures prominently across the literature of several professions. The last common ancestor of "usage-based" and its cognates is the work of the later Wittgenstein, especially the Philosophical Investigations. This suggests that usage-based theories in different disciplines have a set of basic assumptions they share. Still, "usage-based" as a technical term shows subtle differences in meaning in philosophical and nonphilosophical literature. I should say a few words here about those subtle differences.

First, philosophy. In philosophy, usage-based approaches start with the assumption that the meaning of a word is identical with its use. Accordingly, a very simple and popular paraphrase of what use theories of meaning claim is, in effect: meaning is use (see above). Of course, this slogan form of the main axiom obscures many important details of the actual theory. For example, take the 'type vs token' issue that is highly relevant in usage-based considerations (see 8.2). I do not want to go into these niceties here but rather illustrate the broader picture. Roughly, when philosophers say that meaning is use they are making a programmatic claim. What they mean is that, in principle, given enough cognitive resources, memory, and information about the world and its history, it is possible to determine (i.e. to state) the meaning of a given word. Philosophers are not particularly concerned with finding out how a word is actually used. Their programmatic claim is merely meant as a contrast to the view according to which

${ }^{19}$ Other relevant distinctions are 'semantic theories vs foundational theories' (Speaks 2011) or 'theories of semantic content vs theories of communication' (Borg 2004b). 
meanings of words are fixed and can be stated without taking into account particular situations in which language is actually applied. That is to say, usage-based theories are counterparts to theories that approach semantics independently from communication concerns.

What is at stake will become even clearer when I take into account some example cases. ${ }^{20}$ Here is Horwich (1997b), talking this time about vague predicates:

[The use-regularities of vague predicates] do not derive from stipulation, but are implicit in our linguistic practice (insofar as they provide the best explanation of that practice). (933)

And

[...] the explanatorily fundamental regularity in our use of [the vague predicate] " $\mathrm{H}$ " is approximated by a partial function $\mathrm{A}(\mathrm{H})$ which specifies the subjective probability of its applying as a function of the underlying parameter $n$ (i.e. 'number of grains' for "heap", 'number of dollars' for "rich", etc.) [...] Such a use-regularity results from our having learned, regarding various different values of the parameter $n$, that they constitute clear instances of " $\mathrm{H}$ ", somewhat less clear instances, cases in which " $\mathrm{H}$ " is definitely not applied, and slightly less certain cases of inapplicability. [...I]nsofar as $\mathrm{A}(\mathrm{H})$ is really the complete articulation of the basic regularity governing our use of " $\mathrm{H}$ ", then no matter what else is discovered, it cannot imply a confident application of either " $\mathrm{H}$ " or " $-\mathrm{H}$ " to the borderline objects. (933-934, emphasis original)

Again Horwich, this time talking about meaning more generally:

The overall use of each word stems from its possession of a basic acceptance property. For each word there is a small set

${ }^{20}$ The theories listed in the following differ significantly from each other. Here, however, I am currently concerned only with the distinction 'usage-based semantics vs truthconditional, formal semantics'. Keeping this distinction in mind, different theories-e.g. conceptual role semantics (e.g. Block 1986) and the later Wittgenstein-are theoretically on a par, as they all rest on the assumption that meaning is effectively determined by how language is actually applied. 
of simple properties which (in conjunction with other factors and with the basic properties of other words) explain total linguistic behaviour with respect to that word. [...] The present theory is focused on the semantic feature of a word. The distinctive form of that feature is that it designates the circumstances in which certain specified sentences containing the word are accepted; and the primary explanatory role of a word's acceptance property is to account for the acceptance of other sentences containing the word. (1998, 44-45, emphasis omitted)

Greenberg \& Harman (2006, 242), discussing conceptual role semantics, say that

Conceptual Role Semantics (CRS) is the view that the meanings of expressions of a language (or other symbol system) or the contents of mental states are determined or explained by the role of the expressions or mental states in thinking. The theory can be taken to be applicable to language in the ordinary sense $[\ldots]$.

And further down in the same article:

One way to investigate the contribution of use to meaning is to consider how a thinker describes certain imaginary possibilities. For example, one aspect of Mabal's use of concepts is her firm belief that all cats are animals. Other aspects include her firm beliefs that there are cats now, there have been cats in the past, and there will be cats in the future. Another aspect is the way she applies the concept cat to particular things. (305-306)

Block (1986), an early proponent of conceptual role semantics, summarises his view thus:

The internal factor [as opposed to not further specified external factors], conceptual role, is a matter of the causal role of the expression in reasoning and deliberation and, in general, in 
the way the expression combines and interacts with other expressions so as to mediate between sensory inputs and behavioural outputs. A crucial component of a sentence's conceptual role is a matter of how it participates in inductive and deductive inferences. A word's conceptual role is a matter of its contribution to the role of sentences. [...E]lements of language have a total causal role, including, say, the effect of newsprint on whatever people wrap in it. Conceptual role abstracts away from all causal relations except the ones that mediate inferences, inductive or deductive, decision making, and the like. (628)

Wittgenstein, characterising the notion of a language game, asks:

Are "there" and "this" also taught ostensively? - Imagine how one might perhaps teach their use. One will point at places and things, but in this case the pointing occurs in the use of the words too and not merely in learning the use. - [...] Now what do the words of this language signify? - How is what they signify supposed to come out other than in the kind of use they have? And we have already described that. $(2009,9 \mathrm{e})$

And in his Remarks on the Foundations of Mathematics he remarks that

The point of the word "all" is that it admits no exception.True, that is the point of its use in our language; but the kinds of use we feel to be the 'point' are connected with the role that such-and-such a use has in our whole life. $(1967,8 \mathrm{e})$

This cursory list of core aspects of different use theories is supposed to show that no philosopher of language seriously intends to state the properties that constitute meaning. What most use theorists do is specify the 'register', if you like, in which such properties might be articulated best. Horwich likes to have meaning properties articulated in terms of acceptance properties. ${ }^{21}$ Greenberg and Harman consider several possibilities for

${ }^{21}$ Interestingly enough, this motif shines through even when the topic is vagueness (see above). 
narrowing down conceptual roles, one of which is conceivability. Block, who fancies a narrower variant of conceptual role semantics, tries to capture them in terms of inferential relations. Wittgenstein leaves the question entirely open. This list can probably be extended ad libitum. The point is: the commonality between all these different theoretical frames is that they all try to narrow down the all too trivial slogan that 'meaning is use'.

One key aspect of philosophical theories of meaning is that they draw a general, abstract picture of how meaning statements are possible; reallife meaning statements are not of primary concern here. A second key aspect is that, in contrast to linguistic and psychological theories, specifically philosophical theories of meaning are not designed to be especially useful. To borrow Tarski's phrase here, '[semantics] has no pretensions of being a universal patent-medicine for all the ills and diseases of mankind, whether imaginary or real' $(1944,345)$. Theories in formal semantics, for example, are typically meant to be implemented one way or other in computer programs (for the purpose of translation, searchability in semantic nets, voice input, etc.), theories in cognitive grammar are motivated by the search for an integrated theory of cognitive capacities of which language is just one aspect (such that one main purpose is that the theory is compatible with related theories in cognitive science). There is no such practical motivation behind semantic theories in philosophy. ${ }^{22}$ They follow their own, self-referential rules: typical marks of adequacy are naturalism, generality, uniformity, and so on.

Eventually, a third key aspect of usage-based approaches to semantics in philosophy is this: the notion of 'use/usage' employed in these theories is rather abstract, so to say. What philosophers mean when they say that their theory is usage-based is not that they examine uses of a word in order to find out what it means. What a word means is-to philosophers at least-always clear pre-theoretically. In case of doubt, they will tell you what a word means. In this respect, meaning statements in philosophy of language inherit an important feature from the thought experiments in which they appear. In thought experiments, there is no epistemological barrier between the reader and the described situation. In order to find out

${ }^{22}$ The later Wittgenstein is a bit of an exception in this regard, as he stressed the therapeutic aspect of his work. 
what is the case in a given thought experiment, one only needs to read the description carefully. It describes all the relevant facts, or else leaves them unspecified. ${ }^{23}$ Philosophers often construct thought experiments that take a certain semantic interpretation for granted. ${ }^{24}$ Assignment of semantic values often times depends on tacit assumptions in these cases. ${ }^{25}$ The claim that 'meaning is use' is a programmatic or methodological statement: if one wants to find out what a word means, the best option is to scrutinise its use (instead of its alleged reference relations). But the examples that are used to underpin this programmatic claim typically presuppose a specific interpretation that is only valid given certain specific background assumptions.

I shall briefly contrast this notion of "usage-based" with the one employed in linguistics and related fields. When linguists claim their theory is usage-based, what they typically mean is (i) that their research is corpus-driven; (ii) that meaning is a social phenomenon, determined by interacting language users; (iii) that the meaning of a word is constituted by its instances; and (iv) that meaning can only be fully comprehended by considering the situational context of utterances (Tomasello 2003a). To be sure, all this is compatible with more philosophically minded use theories. Philosophical and empirical theories alike originate in Wittgenstein's later work. Yet, linguists' and philosophers' respective interests differ. Proponents of usage-based linguistics emphasise that they utilise 'real' data. i.e. actual use instead of armchair examples. Philosophers, on the other hand, hardly ever need to recur to real conversations in order to qualify as 'usage-based' theorists.

The gist is this: the term "usage-based" (and its cognates) is a technical term both in philosophy and in linguistics. Although there is allegedly a common origin, both terms differ significantly in current theorising. Roughly speaking, in linguistics the term is more or less synonymous to "corpus-driven", i.e. it expresses the idea that language must be scrutin-

${ }^{23}$ Often, most things are specified, because most thought experiments include a clause that says 'this and that is different in the imagined situation; everything else is the same as in the actual world'.

${ }^{24}$ Indeed, there are also a lot of philosophers these days that argue by means of linguistic data (cf. the so-called 'X-Phi movement').

${ }^{25}$ For example, a relatively typical background assumption is that the laws posited by the sciences are, by and large, correct. 
ised in relation to specific situations in which it is applied. In philosophy, "usage-based" is an umbrella term for theories that deviate from the mainstream idea of reducing semantics to reference relations and related notions such as satisfaction, truth, etc. Crucially, though, the two research agendas complement each other. In fact, philosophical theories of meaning can reasonably be conceived as philosophically informed foundations of the empirical theories to which they correspond. 